\title{
Grid-Technology for Chemical Reactions Calculation
}

Gabriel Balint-Kurti ${ }^{1}$, Alexander Bogdanov ${ }^{2}$, Ashot Gevorkyan ${ }^{3}$, Yuriy Gorbachev ${ }^{4}$, Tigran Hakobyan ${ }^{5}$, Gunnar Nyman ${ }^{6}$, Irina Shoshmina ${ }^{2}$, and Elena Stankova ${ }^{2}$

${ }^{1}$ University of Bristol, School of Chemistry, Bristol BS8 1TS, UK

Gabriel.Balint-Kurti@bristol.ac.uk

${ }^{2}$ Institute for High Performance Computing and Information Systems, 199397 St.-Petersburg, Russia

(bogdanov, irena, lena) dcsa.ru

${ }^{3}$ Institute for Informatics and Automation Problems NAS of Armenia, 375014 Yerevan, Armenia

g_ashot@sci.am

${ }^{4}$ St.-Petersburg State Polytechnical University,

195251 St.-Petersburg, Russia

gorbachevacsa.ru

${ }^{5}$ Yerevan Physical Institute,

375036 Yerevan, Armenia

hakob@mail.yerphi.am

${ }^{6}$ Goteborg University,

41296 Goteborg, Sweden

nyman@chem.gu.se

\begin{abstract}
We discuss a possible strategy for implementing a grid-based approach to realizing the immense computational resources required to compute reactive molecular scattering cross sections and rate constants.
\end{abstract}

\section{Introduction}

Evaluation of chemical reaction cross-sections and rate-constants as well as more detailed scattering characteristics is one of the great challenges for computational technologies. The main difficulties are connected with the multi-dimension and multiscale character of the problem. Both the accuracy and the computations of detailed state-to-state scattering cross sections are limited by the availability of computational resources and by the efficiency of the computational algorithm used in their calculation. We examine here a possible solution to these problems through the use of Grid technology. The main concept of Grid technology is the solution of a computational problem through coordinated dynamic resource sharing within a multiinstitutional virtual organizations [1]. Some attempts at such Grid based solutions have already been implemented [2 - 4]. In these cases the Grid infrastructure supports the usage of standard quantum chemistry programs (Gaussian [5] and GAMESS [6]). This paper discusses a possible solution of the reactive scattering problem using a Grid based technology. 


\section{Description of Reactive Scattering Problem}

We consider principally the process $A+(B C)_{\mathrm{m}} \rightarrow(A B)_{\mathrm{n}}+C$ which may take place with or without involving the formation of a quasi-bound or an activated complex $(A B C)^{*}$. When the process occurs via the formation of a quasi-bound complex, both the classical and quantum mechanical treatment of the process show chaotic behavior. The computation of the cross sections and rate constants require large, often unattainably large, amounts of computational resources on a conventional computer. Here we discuss an alternative approach that effectively utilizes Grid technologies.

The problem may be divided into two parts that may be solved separately. The first part being that of calculating the potential energy surface (PES) $V\left(q_{1}, q_{2}, \gamma\right)$ and the second part being the calculation of reaction probabilities and rate constants (or other properties). PESs are usually obtained from $a b$ initio quantum chemical calculations (we consider these results as initial input to our problem), but they are represented in Cartesian co-ordinates that are usually not convenient for further calculations. For the scattering calculations the so-called curvilinear co-ordinates are the most convenient ones to use[7].

One of the computational problems consists in the transformation of the potential energy surface to the new co-ordinates $U\left(x^{0}, x^{1}, x^{2}\right) \equiv V\left(q_{1}, q_{2}, \gamma\right)$. It is also convenient to use certain analytic models for the PESs and to just determine their parameters. The PESs we develop depend on the fairly small number of parameters and these may be optimized to fit different sets of ab initio data [8]. In any case this problem may be solved separately. Since this problem is closely related to the inversion of a large dimensional matrix the corresponding methods may be used for its solution and these algorithms can also be parallelized. This procedure may easily be transferred to a Grid technology, implemented on a Grid of loosely connected computational systems. The calculation of scattering cross sections may be reduced to four sequential problems. The first one is the generation of the classical geodesic equations on the Lagrange surface of body system (at first this method of investigation classical dynamical systems was introduced by Krilov [9]) for the evolution of the system along the reaction coordinate and calculation of trajectory tube distribution; the second one is the problem of the solution Shroedinger equation on geodesic trajectory tubes; the third one is the calculation of the transition $S$-matrix elements and the fourth one is the calculation of transition probabilities $[10,11]$.

\section{Grid for Chemical Reactions Calculation}

The basic principles of the Grid for chemical reaction calculation can be stated as follows: 1) Present computer and data resources as a single virtual environment by developing a web portal on the Grid technology. 2) Build an easy-to-use user web interface for providing access to these resources. 3) Facilitate the sharing of results of research. 4) Organize archiving of input, output, and intermediate data. 
As the basic software for Chemical Grid computing the Globus code will be used because of the following distinguishing advantages [12]. As the scheduling system weplan to use the Nimrod/G tool $[13,14]$, which is designed to manage the computational process including the transfer of input data and of the results of the calculations.

Returning to the problems arising in solving the reactive scattering problem that has been partly discussed in section 3, one must complete the following list of steps:

1) Computation of PES. 2) Parameterization of PES. 3) Solution of geodesic trajectory problems on Lagrange surfaces of the three-body system and the construction of the trajectory tubes distribution. 4) Calculation of the quantum system evolution on the trajectory tubes. 5) Computation of $S$-matrix elements and reaction probabilities for a set of initial phases $\varphi_{s}$ and collision energies $E_{k}^{i}$. 6) Averaging of $S$-matrix element amplitudes over distribution of trajectory tubes, i.e. calculation of probabilities of elementary reactive quantum transitions. 7) Visualization of the results.

A Grid implementation of the solution of the reactive scattering problem implies the establishment of some infrastructure that should include algorithms for the $a b$ initio calculation of PESs and the corresponding databases for different scattering partners. The next step then is the parameterization of the PESs using for instance the nonlinear optimization algorithm described in [9]. The Chemical Grid should contain a number of models that will permit the parameterizations of different aspects of PESs to varying accuracies. The classic geodesic trajectory problem calculation on the Lagrange surfaces for the reactive collision should be implemented for use in a distributed computational environment. Also the quantum reactive scattering part for the different trajectory tubes can be parallelized.

Our experience in complex problem solving using the Grid [15] will be used to achieve these goals. We wish to create a Chemical Grid that will allow the incorporation of both the data banks of different PESs and other properties accumulated by other scientists and our own computational codes.

\section{Acknowledgments}

This work was partly supported by the INTAS grant 03-51-4000 and ISTC A-823.

\section{References}

1. Foster, I, Kesselman, C. and Tuecke, S.: "The Anatomy of the Grid: Enabling Virtual Organisations", International Journal of Supercomputer Applications, 15 (3) 2001, pp. 200-222.

2. Nishikava, T., Nagashima, U. and Sekiguchi, S. "Design and Implementation of Intelligent Scheduler for Gaussian Portal on Quantum Chemistry Grid" In: P.M.A.Sloot, D.Abramson,, A.V.Bogdanov, J.J.Dongarra, A.Y.Zomaya, Yu.E.Gorbachev, eds, Proceedings, Part 3, Computational Science - ICCS 2003, in series Lecture Notes in Computer Science, v. 2659, pp. 244-253, Springer Verlag, ISBN 3-540-40194-6. 
3. Baldrige, K.K., Greenberg, J.P. "Management of Web and Associated Grid Technologires for Quantum Chemistry Computation" In: P.M.A.Sloot, D.Abramson,, A.V.Bogdanov, J.J.Dongarra, A.Y.Zomaya, Yu.E.Gorbachev, eds, Proceedings, Part 4, Computational Science - ICCS 2003, in series Lecture Notes in Computer Science, v. 2658, pp. 111-121, Springer Verlag, ISBN 3-540-40194-6.

4. Sudholt, W., Baldridge, K., Abramson, D., Enticott, C. and Garic, S. "Parameter Scan of an Effective Group Difference Pseudopotential Using Grid Computing", New Generation Computing 22 (2004) 125-135.

5. http://www.gaussian.com

6. https://gridport.npaci.edu/GAMESS

7. Pack, R. T. and Parker, G.A., Quantum reactive scattering in three dimensions using hyperspherical (APH) coordinates. Theory J. Chem. Phys. 87, (1987) 3888.

8. Gevorkyan, A.S., Ghulian, A.V. and Barseghyan, A.R. "Modeling of the Potential Energy Surface of Regrouping Reaction in Collinear Three-Atom Collision System Using Nonlinear Optimization" In: P.M.A.Sloot, D.Abramson, A.V.Bogdanov, J.J.Dongarra, A.Y.Zomaya, Yu.E.Gorbachev, eds, Proceedings, Part 2, Computational Science - ICCS 2003, in series Lecture Notes in Computer Science, v. 2658, pp. 545-554, Springer Verlag, ISBN 3-540-40194.

9. Krylov, N.S., Works by abroad of statistical physics, publishing company Academy of Scince SSSR [in Russian] ed A. Fok, Moscow (1950), p.205

10. Bogdanov, A.V., Gevorkyan, A.S. and Grigoryan, A.G., Bifurcations in trajectory problem as a cause of internal-time singularities and the onset of quantum (wave) chaos, Tech. Phys. Lett. 25, (1999) 637

11. Bogdanov, A.V., Gevorkyan, A.S., Grigoryan, A.G., Internal Time Peculiarities as a Cause of Bifurcations Arising in Classical Trajectory Problem and Quantum Chaos Creation in Three body System, AMS/IP Studies in Advanced Mathematics, V.13. p. 69-80, (1999)

12. Johnston, W. E., The NASA IPG Engineering Team, and The DOE Science Grid Team Implementing production Grid

13. Abramson, D., Giddy, J., Kotler, L.: High Performance Parametric Modeling with Nimrod/G: Killer Application for the Global Grid? International Parallel and Distributed Processing Symposium (IPDPS), Cancun, Mexico (May 2000) 520- 528; http://www.csse.monash.edu.au/ davida/nimrod/

14. Shoshmina, I., Bogdanov, A.V. and Abramson D. "Whither the Grid?" Proceedings of the International Conference "Distributed Computing and Grid Technologies in Science and Education". July 2004, Dubna, Russia. (accepted for publication)

15. Krzhizhanovskaya, V.V., Gorbachev, Yu.E., Sloot, P.M.A. A Grid-based Problem-solving Environment for Simulation of Plasma Enhanced Chemical Vapor Deposition. In: Book of abstracts of the International Conference "Distributed Computing and Grid Technologies in Science and Education". 29 June - 2 July 2004, Dubna, Russia. Publ: JINR, Dubna, 2004. pp.89-90. 